\title{
Leading a programmed death
}

Non-apoptotic cell death is important during animal development, but the genes controlling this type of cell death are unknown. Blum et al. identify pqn-41, which encodes a poly-Glu-repeat protein, as a gene required for linker cell death in Caenorhabditis elegans.

During development of the male reproductive system of C. elegans, the linker cell leads the migration and elongation of the male gonad and then dies between the fourth larval stage and adulthood. Linker cell death is controlled by a cell-autonomous process that does not involve any of the known cell death factors — such as apoptotic caspases and is characterized by specific morphological features; these include indentation of the nuclear envelope, uncondensed chromatin and organelle swelling.

In an RNA interference-based screen for genes required for linker cell death, the authors identified pqn-41 as the only gene, among six clones, exclusively affecting linker cell survival. Surviving linker cells lacking pqn-41 expression did not show any nuclear envelope indentations but did show organelle swelling, indicating that $\mathrm{PQN}-41$ is required for the former but not the latter. Furthermore, inactivation of pqn-41 specifically in linker cells was sufficient for survival, which suggests that PQN-41 functions cell-autonomously.

pqn-41 encodes a protein containing

Glu-rich domains that form six coiled-coil motifs. Protein truncations showed that these coiled-coil regions are necessary for PQN-41 function in promoting cell death. These motifs can be found in prions and in aggregation-prone proteins, which are associated with neurodegenerative diseases. PQN-41 fused to green fluorescent protein formed cytoplasmic aggregates in the linker cell, suggesting that PQN-41 shares structural similarities with those proteins. Importantly, although poly-Glu repeats are often toxic for cells, expression of PQN-41 in cells other than linker cells did not induce death, which indicates that
PQN-41 requires the appropriate cellular context to promote death.

Blum et al. went on to analyse pqn-41 expression and found that it is turned on in linker cells when they begin to die. Furthermore, they identified the mitogen-activated protein kinase kinase SEK-1 as an upstream regulator that is required for pqn-41 expression. Interestingly, the authors observed that the zinc-finger transcription factor LIN-29, which had previously been shown to be involved in linker cell death, does not affect pqn-41 expression. This suggests that SEK-1 and LIN-29 function in parallel, and this was confirmed by the additive effect seen in worms carrying loss-of-function mutations in both genes.

Linker cell death shares morphological similarities with vertebrate developmental cell death, raising the possibility that PQN-41-like proteins might mediate non-apoptotic developmental cell death in vertebrates. In addition, nuclear envelope indentation is seen in poly-Glu expansions underlying neurodegenerative diseases, so it is possible that these proteins might promote neurodegeneration by inappropriately activating a type of cell death similar to that of linker cells.

Kim Baumann

ORIGINAL RESEARCH PAPER Blum, E. S. et al.

Control of nonapoptotic developmental cell death in Caenorhabditis elegans by a polyglutaminerepeat protein. Science 335, 970-973 (2012) 